\title{
Case 2 - A case of secondary platinum-resistant ovarian cancer
}

\author{
Marilena Di Napoli ${ }^{1}$ \\ Commentary: Domenica Lorusso
}

\begin{abstract}
Patients with ovarian cancer relapsing during first-line treatment (refractory) or in the following few months (platinum-resistant) are a very heterogeneous group with various biological tumor behaviors. As this condition is linked to an unfavorable prognosis, the main objective of treatment is to palliate symptoms and preserve quality of life. While traditional chemotherapy may help to achieve this, new biological agents that have been introduced or are under development are expected to improve the quality of life and outcomes for patients with advanced ovarian cancer. This case report describes the clinical history of a 67-year-old woman with bilateral ovarian high grade serous papillary adenocarcinoma with lymph node and omentum metastasis.
\end{abstract}

Key words: advanced ovarian cancer, new biological drugs, quality of life

\section{Introduction}

Despite optimal surgery and appropriate first-line chemotherapy, approximately $70-80 \%$ of patients with epithelial ovarian cancer will develop a disease relapse [1]. The same modalities as used for primary treatment are available for the treatment of recurrent ovarian cancer. Until now, the platinum-free interval has been considered as the main prognostic factor that guides the treatment choice at time of the recurrence. According to this definition, recurrent ovarian cancer has been characterized into four different categories known as platinum-refractory, resistant, partially sensitive, and fully sensitive, depending on when the relapse occurs after the last platinum treatment (during treatment or within 4 weeks; between 6 and 12 months; or beyond 12 months, respectively) [2, 3]. Although these definitions have been used to identify different populations, the resistance to platinum-based treatment is not a categorical variable. Also, all recurrent patients develop secondary resistance over time. Patients relapsing during first-line treat-

${ }^{1}$ Division of Medical Oncology, Department of Uro-Gynecological Oncology, IRCCS, Istituto Nazionale Tumori Fondazione

G. Pascale, Napoli, Italy.

Correspondence to:

Marilena Di Napoli, MD,

Dipartimento di Uro-Ginecologia Oncologica,

Istituto Nazionale Tumori IRCCS Fondazione G. Pascale

Via M. Semmola 52, 80131 Napoli, Italy.

Phone: +390815903637 - Fax: +390815903861

E-mail: marilena.dinapoli@gmail.com

CANCER BREAKING NEWS 2017;5(2):46-50

DOI: $10.19156 / C B N .2017 .0048$ ment (refractory) or in the following few months thereafter (resistant) represent a very heterogeneous group with various biological tumor behaviors. This condition is linked to an unfavorable prognosis, so the main objective of treatment is to palliate symptoms and preserve quality of life. Monotherapy with non-platinum compounds has shown to be equally effective and less toxic than combination therapies. The addition of bevacizumab to single agent nonplatinum chemotherapy prolongs progression-free survival in patients that have not received bevacizumab front line.

\section{Case report}

On April 2006, a 67-year-old woman underwent bilateral salpingo-oophorectomy and omentectomy for bilateral ovarian high grade serous papillary adenocarcinoma with lymph node and omentum metastasis (Figure 1). No family history of cancer was present. After surgery, she underwent chemotherapy with paclitaxel $\left(175 \mathrm{mg} / \mathrm{m}^{2}\right)$ and carboplatin (AUC 5) that induced complete clinical remission. She then started a clinical and radiological follow-up. On May 2010, due to an increase in cancer antigen (CA)-125 serum level (to $450 \mathrm{U} / \mathrm{mL}$ ), a computed tomography (CT) scan showed evidence of multiple abdominal metastasis and ascites. The patient was treated with second-line treatment with pegylated liposomal doxorubicin and carboplatin for 6 cycles, after which she obtained a complete remission (Figure 2). CA-125 levels had returned to baseline levels and remained normal until December 2013. At that time, a BRCA test was performed, and the patient was found to be BRCA1-mutated. Peritoneal carcinomatosis and asci- 


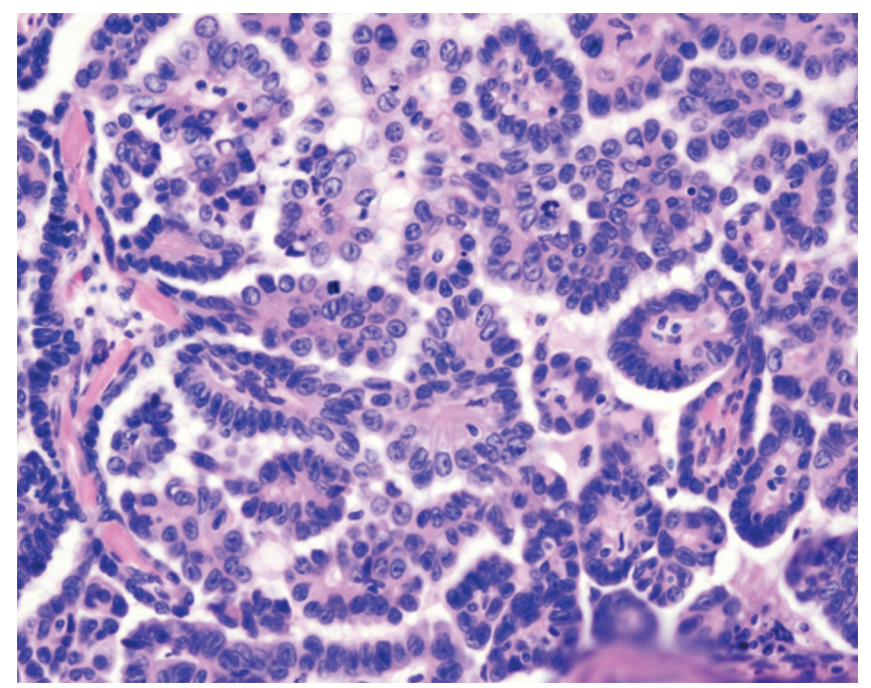

Fig. 1. Histopathological features: serous adenocarcinoma arising in bilateral ovaries.
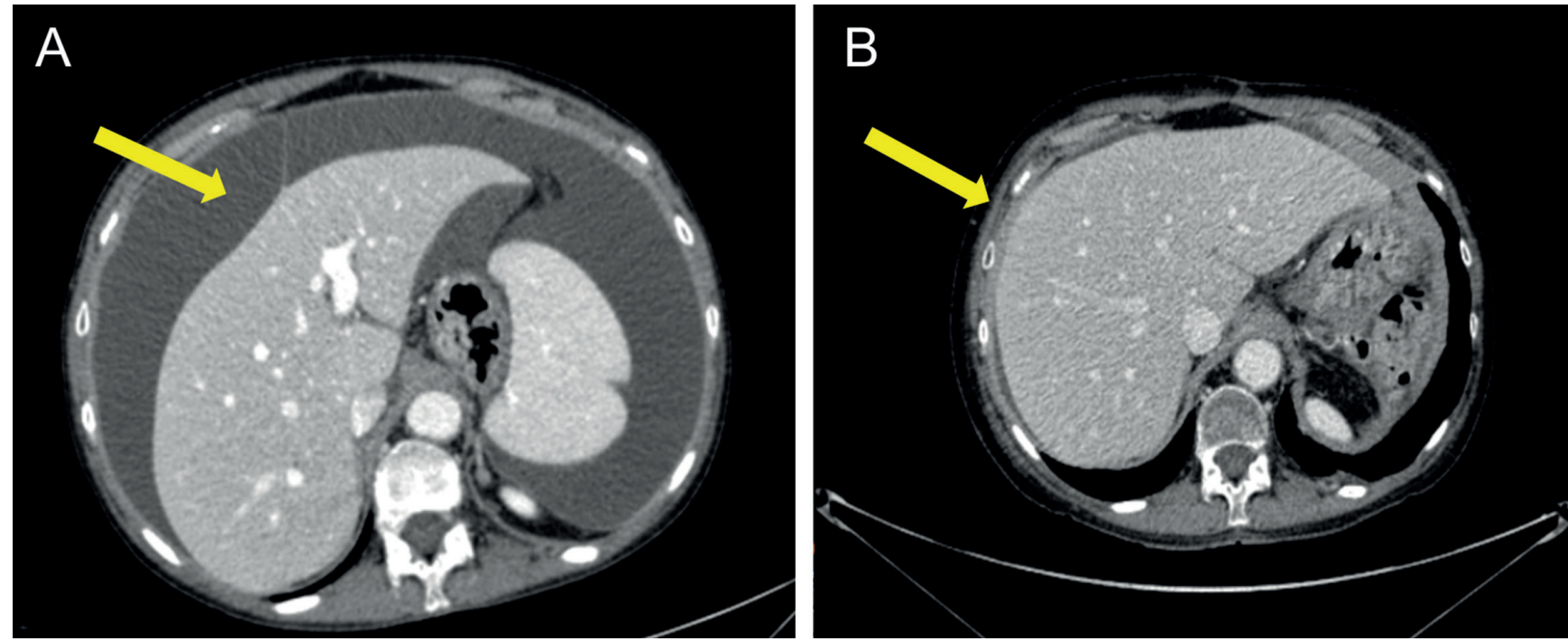

Fig. 2. Computed tomography pre (A) and after (B) second-line treatment (achievement of complete radiological response and absence of ascites).
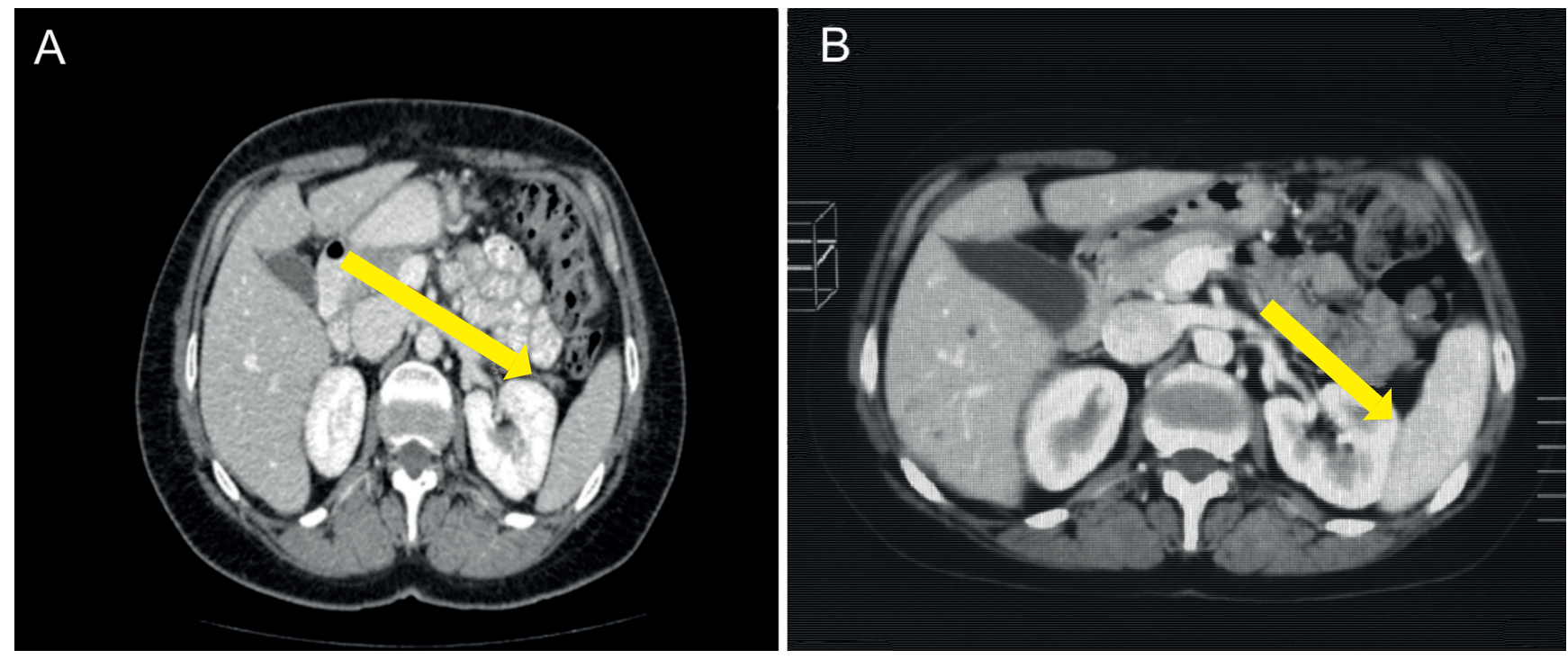

Fig. 3. Presence of peritoneal disease (A) and achievement of complete radiological response after third-line therapy (carboplatin single agent) (B). 
tions were available (weekly paclitaxel and topotecan), the patient refused traditional chemotherapy because of her previous experiences with chemotherapy-related side effects. The patient was referred to a phase 1 center that enrolled her in a trial with an immunotherapy agent. The patient was on treatment with immunotherapy for 10 months and ultimately progressed and died.

\section{Conclusion}

In many patients with recurrent ovarian cancer, the goal of achieving a chronicization of the disease is achievable with traditional chemotherapy. However, the evolution of medical treatments has added new biological agents to our armamentarium, such as bevacizumab and olaparib, that can

\section{References}

1. Siegel RL, Miller KD, Jemal A. Cancer statistics, 2015. CA Cancer J Clin 2015;65(1):5-29.

2. Friedlander M, Trimble E, Tinker A et al. Clinical trials in recurrent ovarian cancer. Int J Gynecol Cancer 2011;21(4):771-5. significantly contribute to this chronicization. Additional agents are under development that will provide further remarkable opportunities for our patients.

\section{Acknowledgments}

The Authors thank Ray Hill, an independent medical writer, who provided native English editing and journal styling on behalf of HPS. This editorial assistance was funded by PharmaMar, Spain.

\section{Conflicts of Interest}

The Authors declare there are no conflicts of interest in relation to this article.

3. McGee J, Bookman M, Harter P et al. Fifth Ovarian Cancer Consensus Conference: Individualized therapy and patient factors. Ann Oncol 2017;28(4):702-10.

\section{Commentary}

Epithelial ovarian cancer is the leading cause of death among all gynecological malignancies. At present, debulking surgery followed by postoperative platinum-based chemotherapy remains the standard first-line treatment of this disease. However, approximately $75 \%$ of patients with advanced ovarian cancer will experience tumor recurrence, and most of them will succumb despite salvage chemotherapies. The 5-year survival of patients with stage III disease and suboptimal residual tumor is about 25\% [1]. The poor outcome of patients with advanced ovarian cancer is mainly due to the lack of effective drugs for relapsed or recurrent diseases which have developed resistance to current chemotherapeutic agents. Thus, the research for effective drugs with pharmacological mechanisms different from conventional therapeutic agents and that do not demonstrate cross-resistance to the initial therapy has become an urgent clinical need. Moreover, given that platinum-resistant recurrent ovarian cancer cannot be completely cured, the treatments have an important role in maintaining the quality of life of the patients [2]. PM01183 (lurbinectedin) is a synthetic tetrahydroisoquinoline that is a selective inhibitor of active transcription [3]. Furthermore, PM01183 affects the inflammatory microenvironment, with selective apoptotic-inducing effect on tumor-associated macrophages, and specific inhibition of inflammatory cytokines production [4]. Strong preclinical antitumor activity was observed in cisplatin-resistant epithelial ovarian cancer models [5].

In a randomized phase 2 trial versus topotecan in 52 patients with platinum-resistant/refractory ovarian cancer lurbinectedin was associated with a $23 \%$ confirmed response, with a median duration of response of 4.6 months and $23 \%$ of responses lasting six months or more. There were no responses in the 29 patients treated with topotecan. Grade $3 / 4$ neutropenia in $85 \%$ of patients, febrile neutropenia in $21 \%$ and fatigue (grade 3 in 35\%) were the main safety findings for PM01183 [6].

Mirvetuximab soravtansine (IMGN853) is an antibody-drug conjugate (ADC) comprising a humanized FRo-bindingmonoclonal antibody conjugated to the cytotoxicmaytansinoideffectormolecule DM4 [7, 8]. IMGN853 binds with high affinity and specificity to FRa on the surface of tumor cells, which, upon antigen binding, promotes ADC internalization and intracellular release of DM4 [9]. DM4 subsequently acts as an antimitotic agent to inhibit tubulin polymerization and disrupt microtubule assembly, resulting in cell-cycle arrest and apoptosis. In preclinical studies, IMGN853 has shown robust antitumor activity in FR $\alpha$-positive tumors, including in models of ovarian cancer [10].

In a phase Ib trial in 46 patients with platinum-resistant epithelial ovarian cancer, presenting FRa 
positivity by immunohistochemistry (>25\% of tumor cells), IMGN853 at $6.0 \mathrm{mg} / \mathrm{kg}$ once every 3 weeks there was a 26\% response rate and a median progression-free survival (PFS) of 4.8 months. Adverse events were generally mild (grade 2), with diarrhea (44\%), blurred vision (41\%), nausea (37\%), and fatigue $(30 \%)$ being the most commonly observed treatment-related toxicities. Grade 3 fatigue and hypotension were reported in two patients each (4\%) [11]. A phase II trial with mirvetuximab soravtansine versus physician's choice chemotherapy in platinum-resistant ovarian cancer patients is ongoing. Target therapy, particularly against vascular endothelial growth factor (VEGF), has been added to chemotherapy for treating ovarian cancer in recent years: in the AURELIA (Avastin Use in PlatinumResistant Epithelial Ovarian Cancer) trial, the median PFS of single-agent chemotherapy with bevacizumab was 6.7 months versus 3.2 months obtained by chemotherapy alone, and the hazard ratio (HR) was 0.48, compared with single agent chemotherapy alone [12]. Unfortunately, due to limitations in reimbursement, bevacizumab is not available in all the European countries.

In the same setting, pazopanib, a tyrosine kinase (TKI) inhibitor with antiangiogenic properties, reported to increase PFS (median 6.35 vs 3.49 months; HR 0.42) when administered in combination with weekly paclitaxel versus weekly paclitaxel alone. The most common grade $3 / 4$ adverse events were neutropenia (30\% in the pazopanib group vs $3 \%$ in the paclitaxel group), fatigue (11\% vs $6 \%)$, leucopenia (11\% vs 3\%), hypertension ( $8 \%$ vs $0 \%$ ), liver toxicity ( $8 \%$ vs none), and anemia (5\% vs $14 \%$ ) [13]. Unfortunately, due to the policy of the pharma company, pazopanib was not further developed for the treatment of ovarian cancer.

In Europe, poly ADP ribose polymerase (PARP) inhibitors have gained label approval as maintenance treatment in patients with platinum-sensitive ovarian cancer, responsive to platinum either BRCA-mutated (olaparib) or regardless of BRCA mutation status (niraparib). On the other hand, in the United States, two PARP inhibitors (olaparib and rucaparib) have been approved as single agent therapy in BRCA-mutated patients, both germline or somatic, who have received at least two (rucaparib) or three (olaparib) previous chemotherapy lines, regardless of platinum sensitivity.

The olaparib conditional approval was based on the results of a single-arm, open-label, pivotal phase II study in which $34 \%$ of 137 patients with germline BRCA-mutated advanced ovarian cancer, who had received three or more prior lines of chemotherapy, had an objective response for a median duration of 7.9 months [14]. Furthermore, based on SOLO2 phase III study, olaparib's new tablet formulation also recently received approval by $F D A$, as maintenance treatment for women with platinum-sensitive recurrent ovarian cancer, regardless of BRCA-mutation status.

Approval for rucaparib was based on a pooled analysis of two phase I and II trials reporting $59.6 \%$ response rate in 108 advanced BRCA-mutated ovarian cancer patients (either somatic or germline) who had received at least two previous chemotherapy lines. The most common treatment-emergent adverse events (all grades) were astheniaffatigue (85.7\%), nausea (83.3\%), anemia (71.4\%), alanine transaminase and/or aspartate transaminase elevations (57.1\%), and vomiting (54.8\%) [15].

More recently, anti-PD-1 antibodies have become a treatment option in ovarian cancer. Hamanishi et al. reported that the response rate and disease control rate with nivolumab in platinum-resistant recurrent ovarian cancer was $15 \%$ and $45 \%$, respectively [16]. Following this pioneer study, several clinical trials of anti PD-L1 and anti-PD-1 antibody are being conducted in all the settings of disease.

Although recurrent ovarian cancer is not a completely curable disease, particularly when the recurrence is praecox, less than six months after completing chemotherapy (platinum-resistant recurrence), the availability of new drugs and new treatment strategies is transforming a rapidly deadly tumor into a chronic disease, allowing our patients to live longer, notwithstanding with the disease. Therefore, the toxicity profile of the drug and the side effects patients experience are very important. Furthermore, it has become mandatory to consider maintenance of an acceptable quality of life for patients as a primary endpoint of clinical research. 


\section{References}

1. Luvero D, Milani A, Ledermann JA. Treatment options in recurrent ovarian cancer: latest evidence and clinical potential. Ther Adv Med Oncol 2014;6:229-39.

2. Ledermann JA, Raja FA, Fotopoulou $\mathrm{C}$ et al, on behalf of the ESMO Guidelines Working Group. Newly diagnosed and relapsed epithelial ovarian carcinoma: ESMO Clinical Practice Guidelines for diagnosis, treatment and follow-up. Ann Oncol 2013;24(Suppl 6):vi24-vi32.

3. Santamaria Nunez G, Robles CM, Giraudon C et al. Lurbinectedin specifically triggers the degradation of phosphorylated RNA polymerase II and the formation of DNA breaks in cancer cells. Mol Cancer Ther 2016;15(10):2399-412.

4. Allavena $\mathrm{P}$, Belgiovine $\mathrm{C}$, Liguori $\mathrm{M}$ et al. Lurbinectedin reduces tumor-associated macrophages and the production of inflammatory cytokines, chemokines, and angiogenic factors in preclinical models [Abstract]. In Proceedings of the 107th Annual Meeting of the American Association for Cancer Research; 2016 Apr 16-20; New Orleans, LA. Cancer Res 2016;76:Abstract nr 1284.

5. Vidal A, Munoz C, Guillen MJ et al. Lurbinectedin (PM01183), a new DNA minor groove binder, inhibits growth of orthotopic primary graft of cisplatin-resistant epithelial ovarian cancer. Clin Cancer Res 2012;18:5399-411.

6. Poveda A, Del Campo JM, Ray-Coquard I et al. Phase II randomized study of PM01183 versus topotecan in patients with platinum-resistant/refractory advanced ovarian cancer. Ann Oncol 2017;28(6):1280-7.

7. Lutz RJ. Targeting the folate receptor for the treatment of ovarian cancer. Transl Cancer Res 2015;4:118-26.

8. Lambert JM. Drug-conjugated antibodies for the treatment of cancer. Br J Clin Pharmacol 2013;76:248-62.

9. Erickson HK, Widdison WC, Mayo MF et al. Tumor delivery and in vivo processing of disulfide linked and thioether- linked antibody-maytansinoid conjugates. Bioconjug Chem 2010;21:84-92.

10. Ab O, Whiteman KR, Bartle LM et al. IMGN853, a folate receptor- $\alpha(\mathrm{FR} \alpha)$-targeting antibody-drug conjugate, exhibits potent targeted antitumor activity against FR $\alpha$-expressing tumors. Mol Cancer Ther 2015;14(7):1605-13.

11. Moore KN, Martin LP, O'Malley DM et al. Safety and activity of mirvetuximab soravtansine (IMGN853), a folate receptor alpha-targeting antibody-drug conjugate, in platinum-resistant ovarian, fallopian tube, or primary peritoneal cancer: A phase I expansion study. J Clin Oncol 2017;35(10):1112-8.

12. Pujade-Lauraine E, Hilpert F, Weber B et al. Bevacizumab combined with chemotherapy for platinum-resistant recurrent ovarian cancer: The AURELIA open-label randomized phase III trial. J Clin Oncol 2014;32(13):1302-8.

13. Pignata S, Lorusso D, Scambia G, MITO 11 Investigators. Pazopanib plus weekly paclitaxel versus weekly paclitaxel alone for platinum-resistant or platinum-refractory advanced ovarian cancer (MITO 11): a randomised, open-label, phase 2 trial. Lancet Oncol 2015;16(5):561-8.

14. Kaufman B, Shapira-Frommer R, Schmutzler RK et al. Olaparib monotherapy in patients with advanced cancer and a germline BRCA1/2 mutation. J Clin Oncol 2015;33:244-50.

15. Kristeleit R, Shapiro GI, Burris HA et al. A Phase I-II Study of the oral PARP inhibitor rucaparib in patients with germline BRCA1/2-mutated ovarian carcinoma or other solid tumors. Clin Cancer Res 2017. doi: 10.1158/1078-0432.CCR-162796. [Epub ahead of print].

16. Hamanishi J, Mandai M, Ikeda T et al. Safety and antitumor activity of anti-PD-1 antibody, nivolumab, in patients with platinum-resistant ovarian cancer. J Clin Oncol 2015;33(34):4015-22. 